\title{
18
}

\section{Analysis of Ignorance Factors in Design Criteria Subject to Model Uncertainty}

\author{
Marc A. Maes \\ Civil Engineering Department, The University of Calgary \\ Calgary, Alberta, Canada T2N 1N4
}

\section{INTRODUCTION}

The effect of model uncertainty on both structural reliability analysis and reliablitybased design has been discussed in a number of articles. Der Kiureghian (1989) and Der Kiureghian and Liu (1986) formulate the basic framework for analysis; they develop several measures of structural safety given imperfect models, and focus on a complete description of the different types and sources of model uncertainty, together with appropriate estimation and analysis methods. Also, several measures of imprecision are proposed.

In a design context, rules and specifications should encourage the gathering of information and the use of more refined models to reduce model uncertainty : this has been investigated by Der Kiureghian (1989) and by Maes (1991), through the use of optimal reliability metrics on the one hand, and through the use of ignorance factors on the other.

In obtaining ignorance factors, an essential objective is to obtain as much information about the behaviour of ignorance factors with varying degrees of uncertainty, on the basis of the smallest possible number of calibration steps. In Maes (1991) a two-step calibration scheme is discussed. The objective of Step 1 is to fine-tune all the partial factors (applicable to the basic random variables) using a reliability-based optimization scheme. This analysis is performed assuming perfect models (zero model uncertainty). Step 2 focuses on determining numerical values of the ignorance factors for varying degrees of model quality, given that the previously derived partial factors remain constant. Ideally, just one analysis would be required to yield optimal design check equations: this is the subject of the present paper.

Winterstein et al. (1994) develop an interesting approach to deriving reliabilitybased design criteria for uncertain models. The idea is to "correct" results based on a median response (which requires an analysis with fixed model uncertainty parameters), based on FORM omission factors. Omission sensitivity factors (Madsen, 1988) give the 
relative error in the reliability index when a basic random variable is replaced by a deterministic number. In this paper, the opposite problem is examined : what is the effect of expanding the analysis by replacing a fixed parameter by a random variable? Inverse FORM is used by Winterstein et al. (1994) to define contours corresponding to a specified level of reliability. This type of analysis is discussed in more detail in Der Kiureghian et al. (1994) and some aspects of it are retained and/or generalized in the present analysis.

\section{MODEL EXPANSION FACTORS}

In this section, we consider a limit state model $g(\mathbf{x}, \overline{\boldsymbol{\theta}})$ for the basic random variables $\mathbf{X}$, formulated using a set of constant model parameters $\overline{\boldsymbol{\theta}}$. These parameters typically originate from a statistical analysis performed in the process of building or fitting the model; or, they could simply represent an empirical estimate or an expert's best opinion. For simplicity, we will assume that $\overline{\boldsymbol{\theta}}$ are the mean values of the random variables $\Theta$ to be considered next. This assumption is not restrictive : a simple scaling of the subsequent results needs to be performed if they are different from the means of $\Theta$.

Let $P_{0}$ be the failure probability associated with the failure region $\{g<0\}$ where we keep in mind that $g$ is a deterministic model with constant parameters $\overline{\boldsymbol{\theta}}$ :

$P_{0}=\operatorname{Pr}(g(\mathbf{X}, \overline{\boldsymbol{\theta}})<0)$

This result is now contrasted with an "expanded" structural reliability analysis which includes all model errors and parameters in the set of random variables $\Theta$ :

$P_{m}=\operatorname{Pr}(g(\mathbf{X}, \boldsymbol{\Theta})<0)$

The basic objective of the "expansion" problem considered here is to estimate $P_{m}$ using information from the $P_{0}$ analysis only, i.e. the analysis without model uncertainty. This problem may be contrasted with the "omission" problem (Madsen, 1988), where the relative error on the reliability index is estimated when one or more variables are replaced by deterministic number(s) : this would correspond with the converse problem of finding $P_{0}$ based upon a full $P_{m}$-analysis.

It is clear that $P_{m} \rightarrow P_{0}$ as $\Theta$ converges in distribution to the fixed set $\overline{\boldsymbol{\theta}}$. Furthermore, we have :

$P_{m}(\mathbf{X}, \Theta)=\int_{\theta} P(\mathbf{X} \mid \boldsymbol{\theta}) f_{\theta}(\boldsymbol{\theta}) d \boldsymbol{\theta}$

where $P(\mathbf{X} \mid \boldsymbol{\theta})$ stands for $\operatorname{Pr}(g(\mathbf{X}, \boldsymbol{\theta})<0)$ conditional upon $\boldsymbol{\Theta}=\boldsymbol{\theta}$, and $f_{\theta}(\boldsymbol{\theta})$ is the joint density of $\boldsymbol{\theta}$. A Taylor expansion of the integrand about the mean vector $\overline{\boldsymbol{\theta}}$, followed 
by integration, yields a "model expansion factor" $\xi_{\theta}$, approximately equal to:

$\xi_{\theta}=\frac{P_{m}}{P_{0}} \cong\left[1+\frac{1}{2} \sum_{i} \sum_{j} \frac{1}{P_{0}}\left(\frac{\partial^{2} P}{\partial \theta_{i} \partial \theta_{j}}\right)_{\theta=\bar{\theta}} \sigma_{\theta_{i} \theta_{j}}\right]$

where $\sigma_{\theta_{i} \theta_{j}}$ are the elements of the covariance matrix $\boldsymbol{\Sigma}_{\theta \theta}$ of the model uncertainties $\Theta$. Exact values of the second order sensitivities may be hard to obtain under general conditions. However, as shown in the next section, excellent asymptotic estimates can readily be obtained as a by-product of the basic $P_{0}$-analysis.

\section{ASYMPTOTIC EXPRESSIONS FOR THE EXPANSION FACTOR}

Asymptotic expressions for first-order parameter sensitivities of $P(\mathbf{X}, \boldsymbol{\theta})$ can be found in Breitung (1994). Breitung's analysis covers both distributional parameters as well as model parameters. Here, only the latter are required, namely :

$\frac{\partial P(\mathbf{X}, \boldsymbol{\theta})}{\partial \theta_{i}} \sim-P_{0}\left[\frac{\partial g}{\partial \theta_{i}} \frac{|\nabla l|}{|\nabla g|}\right]_{\theta=\bar{\theta}, \mathbf{x}=\mathbf{x}^{*}}$

where the gradient $\boldsymbol{\nabla}$ is taken with respect to $\mathbf{x}$, and where $\mathbf{x}^{*}$ represents the coordinates (in the original variable space) of the point of maximum likelihood (PML), as described in Breitung (1994) and Maes et al.(1993); the PML $\mathbf{x}^{*}$ can readily be obtained as the solution of the basic optimization problem needed to solve the structural reliability analysis problem for the $P_{0}$-case, i.e. to maximize $l(\mathbf{x})$ subject to $g(\mathbf{x} \mid \overline{\boldsymbol{\theta}})=0$, where $l(\mathbf{x})=\ln f_{\mathbf{x}}(\mathbf{x})$ is the loglikelihood function of the basic random variables. Breitung's (1994) sensitivity factor analysis is based on a generalization of Leibnitz' rule for the derivative of parameter-dependent integrals, and on asymptotic expansions for multivariate Laplace type integrals. This analysis can easily be extended (Breitung, $1994 \mathrm{~b}$ ) to second-order sensitivities, which yields the asymptotic approximation of the "expansion" ratio (4):

$\frac{P_{m}}{P_{0}} \sim 1+\frac{1}{2}\left[\left(\frac{\nabla l}{\nabla g}\right)^{2}\left(\nabla_{\theta} g\right)^{T} \boldsymbol{\Sigma}_{\theta \theta} \nabla_{\theta} g\right]_{\bar{\theta}, \mathbf{x}^{*}}$

It should be stressed that the ratio $|\nabla l| /|\nabla g|$ is equal to the Lagrange multiplier associated with the above optimization problem.

\section{IGNORANCE FACTORS}

\subsection{Comparing Basic Design Checks With and Without Model Uncertainty}

First, consider the perfect model without model uncertainty. Assume that the mathematical expression used for the deterministic design check (e.g. in LRFD format) is the 
same as that of the limit state model (They do not strictly need to be the same for the subsequent analysis to apply, but the notation simplifies considerably).

Therefore, in order to determine the (minimum) required resistance, denoted here by some capacity-related design parameter $r_{0}$, the following DCE needs to be solved :

$g\left(\mathbf{x}^{*}, \overline{\boldsymbol{\theta}}, r_{0}\right)=0$

where $\mathbf{x}^{*}$ are the (input) design values of the basic variables $\mathrm{X}$. This DCE entirely defines the corresponding limit state model $g\left(\mathbf{X}, \overline{\boldsymbol{\theta}}, r_{0}\right)$. For this model, of course, we wish to achieve a desired reliability level, i.e.

$P\left(\mathbf{X}, \overline{\boldsymbol{\theta}}, r_{0}\right)=P_{T}$

where the same abreviation is used for $P$ as in (3), and where $P_{T}$ denotes the target failure probability. If the model without model uncertainty is perfectly calibrated, $\mathbf{x}^{*}$ may, without loss of generality, be considered to correspond to the PML on the surface (7).

The second step is now to include model uncertainty in the reliability analysis. The model $g$ is mathematically the same. A larger resistance $r_{m}$ will now be required to meet the same target reliability level :

$P\left(\mathbf{X}, \boldsymbol{\Theta}, r_{m}\right)=P_{T}$

At the design level, however, it makes sense to keep things simple; the approach is :

- to keep the same design values of the basic variables; the load and resistance factors, the specified probability levels, etc..., used in (7) remain unchanged.

- to use ignorance factors $\boldsymbol{\theta}^{*}$ to encapsulate the effect of model uncertainty (Maes, 1991).

This results in the following DCE :

$g\left(\mathbf{x}^{*}, \boldsymbol{\theta}^{*}, r_{m}\right)=0$

If (10) is linearized with respect to $\boldsymbol{\theta}$ and $r$ in the neighbourhood of $\overline{\boldsymbol{\theta}}$ and $r_{0}$, and after inserting (7), it follows that :

$\left(r_{m}-r_{0}\right) \cong\left[-\left(\frac{\partial g}{\partial r}\right)^{-1}\left(\nabla_{\theta} g\right)^{T}\left(\boldsymbol{\theta}^{*}-\overline{\boldsymbol{\theta}}\right)\right]_{\mathbf{x}^{*}, \bar{\theta}, r_{0}}$

The essential aspect of the design rule (10) is that $\mathbf{x}^{*}$ is unchanged from (7). Consequently, the original DCE (7) can be used to achieve (9), provided ignorance factors are used in (7) rather than mean values. The following section shows that approximate $\boldsymbol{\theta}^{*}$ can be determined solely on the basis of a $P_{o}$-analysis. 


\subsection{Inverse Reliability}

Given that a properly calibrated model (8) is available using the fixed parameters $\overline{\boldsymbol{\theta}}$, the next step is now to ensure that, by adjusting the ignorance factors in (10), the inclusion of model uncertainty also results in a model having the desired level of reliability (9). A Taylor expansion of $P_{m}$ yields :

$P\left(\mathbf{X}, \Theta, r_{m}\right) \sim P\left(\mathbf{X}, \Theta, r_{0}\right)+\left(r_{m}-r_{0}\right) \frac{\partial P\left(\mathbf{X}, \Theta, r_{0}\right)}{\partial r}$

The "expansion" result (4) may now be used to link the $P_{m}$ and $P_{0}$ analyses at $r=r_{0}$; when higher order derivatives are neglected, and when $\left(r_{m}-r_{0}\right)$ is replaced by (11), together with the condition

$P\left(\mathbf{X}, \Theta, r_{m}\right)=P\left(\mathbf{X}, \overline{\boldsymbol{\theta}}, r_{0}\right)=P_{T}$

then the asymptotic version of (12) can be derived based on (6) and (5):

$\left(\nabla_{\theta} g\right)^{T}\left(\boldsymbol{\theta}^{*}-\overline{\boldsymbol{\theta}}\right) \sim-\frac{1}{2} \frac{|\nabla l|}{|\nabla g|}\left(\nabla_{\theta} g\right)^{T} \Sigma_{\theta \theta} \nabla_{\theta} g$

and, for the special case of just one ignorance factor:

$\theta^{*} \sim \bar{\theta}-\frac{1}{2} \frac{|\nabla l|}{|\nabla g|} \frac{\partial g}{\partial \theta} \sigma_{\theta}^{2}$

with all of the derivatives evaluated at the PML of the $P_{0}$-problem. In the single parameter case, the magnitude of $\left(\theta^{*}-\bar{\theta}\right)$ is thus seen to be proportional to the variance of the model uncertainty (see, for example, Maes, 1991).

The previous approach can be extended to multiplicative uncertainties. This leads to a design format which is quite pervasive in all areas of civil engineering. The asymptotic expression for an ignorance factor $\psi^{*}$ associated with a (single) multiplicative model uncertainty $\Psi$, for which :

$\Psi>0 \quad$ and $\quad \boldsymbol{E}(\Psi)=1$,

can most conveniently be derived from the previous results using a logarithmic transformation $\varphi=\ln \psi$, together with an adjustment for $\mathbf{E}(\ln \psi)$ in the above equations

$\psi^{*}=1-\frac{1}{2} v_{\psi}^{2}\left(\frac{|\nabla l|}{|\nabla g|} \frac{\partial g}{\partial \psi}+1\right)_{\psi=1, \mathbf{x}=\mathbf{x}^{*}}+o\left(v_{\psi}^{2}\right)$

where $v_{\psi}$ is the coefficient of variation $(\mathrm{COV})$ of the model uncertainty parameter $\Psi$; the PML $\mathbf{x}^{*}$ is obtained for the model $g(\mathbf{x} \mid \psi=1)$ with mean model uncertainty 1 . 


\section{EXAMPLE APPLICATION}

Collapse of downhole oil and gas casing and tubing structures occurs when a pipe is accidentally or intentionally evacuated of internal fluids. As a result, the thick-walled tubular is exposed to the full external pressure induced by the formation pore pressure. The sensitivity of the collapse failure mode to imperfections, especially when the onset of plasticity precedes instability, makes it difficult to "predict" collapse loads. In a major development of reliability-based criteria for casing and tubing pipes (Gulati et al., 1994), the ultimate capacity of moderately thick and thick tubes loaded by external pressure is, therefore, calibrated based on the results of well executed experiments. Several data sets are available and they show different degrees of uncertainty depending on manufacturer, type of use, grade, age, geographical and geological context, etc...

Regression allows the COV of the multiplicative model uncertainty $\Psi$ to be determined on the basis of a comparison of each series of test results with the analytical expression developed by Timoshenko and Gere(1961); the objective is then to determine ignorance factors for this model in order to allow for easy consideration of any degree of model quality and variability. The idea is thus to compensate for increasing model error by means of "reducing" the nominal collapse capacity using appropriate ignorance factors $\psi^{*} \leq 1$. The limit state model contains 5 basic random variables and one model uncertainty variable $\Psi$ :

$g\left(\sigma_{y}, E, t, \xi, \triangle p, \Psi\right)=\Psi \cdot p_{c}\left(\sigma_{y}, E, t, \xi\right)-\triangle p$

where $\Delta p$ is the net internal-external pressure difference at a point along the casing string, and $p_{c}$ is the Timoshenko collapse capacity :

$p_{c}\left(\sigma_{y}, E, t, \xi\right)=\frac{1}{2}\left[p_{Y}+p_{e}\left(1+\frac{3 \xi D}{t}\right)-\sqrt{\left(p_{Y}+p_{e}\left(1+\frac{3 \xi D}{t}\right)\right)^{2}-4 p_{Y} p_{e}}\right]$

where $D$ is the outer diameter, and $t$ is the wall thickness. The ovality $\xi$ is defined as $\xi=\frac{2\left(D_{\max }-D_{\min }\right)}{D_{\max }+D_{\min }}$. The elastic buckling pressure is $p_{e}=\frac{2 E}{1-\nu^{2}}\left(\frac{t}{D}\right)^{3}$ and the yield pressure is $p_{Y}=2 \sigma_{y} \frac{t}{D}$, where $\sigma_{y}$ is the yield stress, $E$ is Young's modulus, and $\nu$ is Poisson's ratio.

The effect of $\Psi$ depends critically on the variability of the "loading term" $\Delta p$ in (18). Several load domains were considered in the study (Gulati et al., 1994) as part of the overall zonation scheme of the design set. As an example, we only consider salt loading (direct contact with a flowing salt formation), which imposes the most severe collapse load on casing. Also, the present analysis is restricted to just one nominal $D / t$ ratio $(D / t=12)$, one steel grade (L80) (In reality, a wide range of conditions were assumed).

It suffices then to calibrate the nominal values in (18) in such a way that $\operatorname{Pr}(g \leq$ $0)=P_{T}=10^{-3.5}$. This analysis is performed with $\Psi$ set to one, that is, with the 
probability distributions of the 5 basic variables only. The PML and the Lagrange multiplier $\lambda=|\nabla l| /|\nabla g|$ are obtained automatically, and together with the fact that $\frac{\partial g}{\partial \psi}=p_{c}$ at the PML, equation (17) can be used directly to determine the required ignorance factor $\psi^{*}$ as a function of the Timoshenko model error $\mathrm{COV}, v_{\psi}$.

The resulting ignorance factor is shown in Figure 1. It can be seen that a model uncertainty $\mathrm{COV}$ of $10 \%$ requires an ignorance factor $\psi^{*}$ of about 0.87 . This indicates that, for the design conditions considered in this example, a $87 \%$ reduction of the collapse capacity $p_{c}$ would be needed to achieve a design product with the same reliability level as that corresponding with the use of a perfect model without experimental error.

For comparison, Figure 1 also shows "exact" values of $\psi^{*}$. These were obtained by including a lognormal random variable $\Psi$ with a COV varying in the range 0 to 0.15 , and solving each resulting 6 -variable inverse reliability problem to the target value $P_{T}=10^{-3.5}$; following (18) the "exact" ignorance factor associated with a model error $\mathrm{COV}$ equal to $v$ is then given by the ratio $p_{c}\left(v_{\psi}=0\right) / p_{c}\left(v_{\psi}=v\right)$. It can be seen that the values provided by the expansion approach are indeed $o\left(v_{\psi}^{2}\right)$ and that, in this case, they slightly overestimate the required collapse resistance reduction, which makes for a somewhat conservative design rule.

\section{CONCLUSIONS}

Model expansion factors are useful in assessing the effect of model uncertainties on a reliability analysis performed on the basis of an imperfect model. They address the question of how the failure probability $P_{m}$ varies with respect to the base case of a perfect model $\left(P_{0}\right)$, if model uncertainty variables are included in the analysis. It is shown that the ratio $P_{m} / P_{0}$ can be approximated using information from a $P_{0}$-analysis only.

Ignorance factors intervene in the inverse problem of reliability-based design. For this second type of problem, the critical question is how the design check equations need to be modified in order to "compensate" for the effect of increasing model uncertainty.

In both cases, the factors can be determined on the basis of an analysis which ignores model uncertainty (i.e. constant mean values of $\Theta$ and $\Psi$ ). In fact, only the Lagrange multiplier $|\nabla l| /|\nabla g|$ of the constrained loglikelihood maximization problem needs to be determined together with the gradient of $g$ with respect to $\boldsymbol{\theta}$ a the resulting maximum point. The approximations result in accurate failure probability ratio estimates, and in reliable error-inclusive design rules.

\section{REFERENCES :}

Breitung, K (1991). "Probability approximations by loglikelihood maximization", J. Engrg. Mech., ASCE, 117(3), 457-477. 
Breitung, K (1994). Asymptotic approximations for probability integrals. Mathematics Series, Springer Lecture Notes in Mathematics, No. 1592.

Breitung, K (1994b). "Parameter dependent integrals : some mathematical tools", 6th IFIP Working Conference on Reliability and Optimization of Structural Systems, Assisi (Perugia), Italy.

Der Kiureghian A. and Liu, P.-L. (1986). "Structural reliability under incomplete probability information", J. Engrg. Mech., ASCE, 112(1), 85-104.

Der Kiureghian A. (1989). "Measures of structural safety under imperfect states of knowledge", J. Struct. Engrg., ASCE, 115(5), 1119-1140.

Der Kiureghian A., Zhang, Y., and Li, C.-C. (1994). "Inverse reliability problem", J. Engrg. Mech., ASCE, 120(5), 1154-1159.

Gulati, K.C., McKenna, D.L., Maes, M.A., Brand, P.R., Johnson, R.C., Lewis, D.B., Riekels, L. and Maute, R.E. (1994). "Reliability-based design and application of drilling tubulars", 1994 Offshore Technology Conference, Houston, No. 7557, 4, 423-430.

Madsen, H.O. (1988). "Omission sensitivity factors", Structural Safety, 5, 35-45.

Maes, M.A. (1991). "Codification of design load criteria subject to modeling uncertainty", J. Struct. Engrg., ASCE, 117(10), 2988-3007.

Maes, M.A., Breitung, K. and Dupuis, D.J. (1993). "Asymptotic importance sampling", Structural Safety, 12, 167-186.

Timoshenko, S.P. and Gere, J.M. (1961). "Theory of elastic stability. McGraw Hill Brook Cy., New York.

Winterstein, S.R., Ude T.C., Cornell, C.A., Bjerager, P., and Haver, S. (1994). "Environmental parameters for extreme response : inverse form with omission factors", Structural Safety $\&$ Reliability, Ed. by Schüeller, G.I., Shinozuka, M. and Yao, J.I.P., Vol.I, 551-557.

Figure 1: Ignorance Factor For The Timoshenko Collapse Limit State

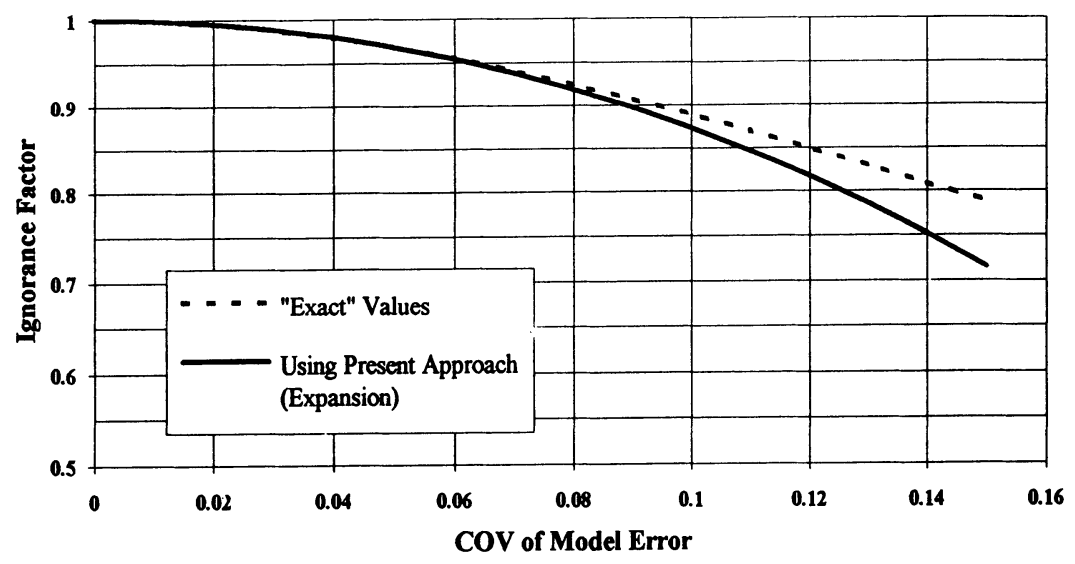

